\title{
No me banco la gente así. Un estudio comparativo del uso de palabras coloquiales y malsonantes en conversaciones informales en tres comunidades de habla hispanohablantes
}

\author{
Eli-Marie D. Drange (Universidad de Adger, Noruega)
}

\begin{abstract}
The present article aims at describing and comparing the use of slang words and swearwords in teenage talk from three different Spanish speaking communities. The analysis builds on spontaneous teenage conversations from the corpus of teenage language Corpus Oral de Lenguaje Adolescente (COLA). This corpus contains informal conversations from Madrid, Buenos Aires and Santiago de Chile; and for this study one conversation from each city has been selected. The aim of the analysis is twofold, in the first place to describe and compare the slang words and swearwords registered in the three conversations, and secondly to study the pragmatic functions of these words. The analysis of the slang words shows that there is variation in the informal words and expressions used in the three communities, and that these expressions seem to be mostly regional.

Regarding the swearwords used, there are similarities in the three communities. There are also similarities in the pragmatic functions of the slang words and swearwords in context. The conclusions that can be drawn from the analysis, is that the Spanish speaking communities have a common system regarding how the slang words are used, but each community uses their creativity in developing words and expressions. For swearwords, the system and the words used are more similar, which might indicate that the communities share the cultural aspects that are reflected in the swearwords.
\end{abstract}

Keywords: Slang words, swearwords, corpus linguistics, teenage talk, Spanish dialects

\section{Introducción}

El objetivo del presente artículo es describir el uso de palabras coloquiales y malsonantes en conversaciones informales en tres comunidades de habla hispanohablantes. El estudio del español hablado ha aumentado las últimas décadas, especialmente gracias a la creación de corpus orales como PRESEEA (Moreno Fernández 2005) y Val.es.co (Pons Bordería y Ruiz Gurillo 2005). El material usado en este artículo también se basa en un corpus oral, el Corpus Oral del Lenguaje Adolescente (COLA), recogido y preparado por la Universidad de Bergen (Jørgensen 2007) ${ }^{1}$

\footnotetext{
${ }^{1}$ http://colam.org/index-espannol.html
} 
El corpus COLA consiste en conversaciones informales de jóvenes de Madrid, Buenos Aires y Santiago de Chile, y en este artículo se analizará una conversación de cada metrópolis. En primer lugar, se presentará la forma de las palabras coloquiales y malsonantes encontradas en las conversaciones. En segundo lugar, se procederá a analizar las palabras en contexto, es decir se realizará un análisis de su función pragmática. El artículo concluirá con una comparación y una discusión sobre las diferencias y similitudes en el uso de palabras coloquiales y malsonantes en las tres comunidades de habla.

\section{Definiciones y limitaciones}

La oralidad se manifiesta de diferentes maneras y varía según las características de los hablantes y la situación de la comunicación, donde la situación de la comunicación puede ser clasificada como más (+) o menos (-) formal (Briz Gómez 2001, 25-26). Además, las intervenciones en un intercambio oral pueden variar desde intervenciones dialogadas a intervenciones más bien monologadas (Calsamiglia Blancafort y Tusón Valls 2007, 19). En el corpus COLA, la mayoría de las conversaciones se pueden clasificar como conversaciones coloquiales siguiendo la definición de Pons Bordería y Ruiz Gurillo: "intercambios caracterizados por no tener una toma de turno predeterminada y cuyos hablantes se expresen con un nivel de formalidad mínimo, independientemente de su procedencia social" (Pons Bordería y Ruiz Gurillo 2005, 243-44). Un grado de formalidad mínimo y una relación de proximidad estrecha entre los participantes constituyen condiciones ideales para la creatividad léxica y el uso de un vocabulario coloquial y malsonante (Briz Gómez 2001), condiciones que hacen del corpus COLA un material ideal para el estudio del uso de palabras coloquiales y malsonantes en contexto.

Si bien existen algunos estudios sobre las palabras tabú (Stenström 2006) (Calvo Shadid 2009) (Gómez Molina 2003) no es un tema tratado exhaustivamente en el contexto hispánico. El acceso al registro informal es un requisito necesario para poder realizar un estudio más extenso que una recopilación de vocabulario coloquial o malsonante. No obstante, la clasificación de un vocablo como coloquial o malsonante no siempre resulta evidente. El Diccionario de la lengua española en la red $^{2}$ (Real Academia Española) (DRAE) define una palabra 'coloquial' como "propio de una conversación informal y distendida", mientras una expresión clasificada como 'malsonante' es una expresión "Que ofende al pudor, al buen gusto o a la religiosidad". Ahora bien, una palabra puede ofender al pudor de un hablante, al mismo tiempo que es de uso común para otro. Nos movemos entonces por un terreno sin límites claros, donde no se puede impedir cierta subjetividad. Para ilustrar la complejidad del tema, sirve como ejemplo una comparación entre la clasificación de la palabra 'teta' en el DRAE y el diccionario de María Moliner (2007). Cuando el DRAE clasifica la palabra 'teta' como "1. f. mama (\| órgano glanduloso)", el diccionario de María Moliner clasifica 'teta' como informal cuando se usa para animales y como vulgar cuando se usa en referencia a la mujer (Moliner 2007).

Un estudio del léxico se puede realizar con diferentes objetivos: la lexicología y la dialectología comparten el objetivo de recoger y documentar las palabras existentes en una comunidad de habla. Cuando la lexicología utiliza el léxico documentado como fuente para elaborar diccionarios, la dialectología se interesa por limitar la extensión de las palabras desarrollando atlas lingüísticos. A diferencia de la dialectología, la sociolingüística se interesa

\footnotetext{
${ }^{2}$ http://www.rae.es/ Las búsquedas se han realizado en la edición de 2017 el 3 de marzo de 2018.
} 
por la distribución diastrática de las palabras, estudiando la variación léxica en relación con variables sociales. Según Hernández Campoy $(1993,176)$ y basándose en Chambers y Trudgill (1980), la geolingüística es una confluencia de la geografía lingüística, la dialectología urbana y la geografía humana, es decir una combinación entre la dialectología tradicional y la sociolingüística.

El acercamiento al léxico informal y malsonante adoptado en este artículo se inspira en la geolingüística al documentar y comparar las palabras coloquiales y malsonantes usadas en conversaciones informales en tres comunidades hispanohablantes diferentes: Madrid, Buenos Aires y Santiago de Chile. El estudio del léxico no destaca en estudios comparativos como los realizados por PRESEEA, que ha definido los estudios de la /d/ intervocálica, la expresión de sujeto, el uso del verbo haber y la atenuación como sus temas prioritarios $\left(\right.$ PRESEEA $^{3}$ ) (Malaver y Samper Padilla 2017).

Además de la comparación geolingüística, el artículo pretende realizar un análisis discursivo basado en corpus (Stenström 2006, 117) profundizando en las funciones pragmáticas de la palabra en contexto. Para estudiar la función pragmática, partimos de la pragmalingüística y de que las funciones comunicativas de los enunciados son estrategias utilizadas por el hablante para hacer llegar su mensaje al interlocutor (Briz Gómez 2001, 1056). Se puede distinguir entre una variedad de funciones pragmáticas, entre las que Briz Gómez (2001, 106) resalta los conectores, intensificadores y atenuadores, además de los marcadores metadiscursivos. Entre estos últimos, Briz Gómez (2001, 224-25) sostiene que predominan los que cumplen una función expreso-apelativa y fática en la conversación coloquial. En el lenguaje juvenil, esta función se cumple en gran medida por vocativos (Jørgensen y Drange 2011). Por la naturaleza de las palabras coloquiales y malsonantes, las categorías más relevantes para este estudio son los marcadores metadiscursivos, los conectores y los intensificadores.

Las palabras malsonantes también se pueden clasificar en diferentes categorías pragmáticas, como las descritas por Ljung (2011). El estudio de las funciones pragmáticas de insultos relacionados con la madre realizado por Hasund, Drange y Stenström (Hasund, Drange, y Stenström 2014; Drange, Hasund, y Stenström 2014) indica que la función pragmática más común en el español hablado en Madrid es la llamada 'name-calling' por Ljung. En este estudio, la única expresión usada en esta categoría es "hijo de puta", que según Gómez Molina $(2003,646)$ es el insulto más frecuente en español. La categoría 'namecalling' se refiere entonces a expresiones negativas dirigidas directamente al interlocutor o a un tercero, y pueden ser tanto vocativos como insultos.

En el presente artículo, la combinación entre la documentación geolingüística y el análisis discursivo y pragmático ayudará a determinar con mayor grado de seguridad si un término específico que existe en varias comunidades de habla también se usa de la misma manera en las diferentes comunidades de habla.

\footnotetext{
${ }^{3}$ PRESEEA. "Metodología" Accedido el 5 de julio de 2017, de http://preseea.linguas.net/Metodolog\%C3\%ADa.aspx
} 


\section{Metodología}

\subsection{Corpus de estudio}

Como se ha mencionado en la introducción, el material analizado en este artículo se ha extraído del corpus COLA. Este corpus recoge conversaciones entre jóvenes de tres comunidades de habla hispanohablantes: Madrid, Buenos Aires y Santiago de Chile. Para el presente artículo se ha seleccionado una conversación de alrededor de 1000 palabras de cada comunidad. Las tres conversaciones son de chicos y chicas de clase media, para tener un material más o menos comparable. Los detalles de los tres archivos se pueden apreciar en Tabla 1:

\begin{tabular}{llll} 
Comunidad & Madrid & Buenos Aires & Santiago de Chile \\
\hline Archivo & MALCE2-3A & BABAS4-1-1 & SCAW4 -03 \\
\hline Participantes & chicos y chicas & chicas & chicos y chicas \\
\hline Nivel social & clase media & clase media & clase media \\
\hline Total palabras & 950 palabras & 1200 palabras & $\begin{array}{l}\text { total } 865 \text { palabras } \\
\text { p. 1-7 (1. 209) (el archivo } \\
\text { es mucho más grande) }\end{array}$ \\
\hline
\end{tabular}

Tabla 1: Detalles del corpus extraído para el presente artículo.

Además, se han realizado algunas búsquedas en la totalidad de los corpus COLAm de Madrid, COLAba de Buenos Aires y COLAs de Santiago de Chile para aportar más información sobre la distribución y el uso de algunas palabras.

\subsection{Selección y organización de los datos}

Para determinar si una palabra es coloquial o malsonante, se ha consultado el Diccionario de la lengua española en la $\operatorname{red}^{4}$ (DRAE). Para las palabras que no se han encontrado en el DRAE, se ha recurrido a otros diccionarios regionales como Diccionario lunfardo del hampa y el delito de Raúl Tomás Escobar y Diccionario etimológico del lunfardo de Oscar Conde, entre otros. El diccionario funciona como un criterio objetivo para la clasificación de las palabras, pero esto no significa que los hablantes de la lengua estén de acuerdo con la clasificación de una palabra como normal o como malsonante. Según Manuel Seco (2003) al tratar el proceso de incorporación de vocabulario americano al DRAE "los riesgos de error son frecuentes e inevitables." (Seco 2003, 376). Así el punto de partida para la clasificación es el DRAE, pero se recurre a otros diccionarios y al análisis del contexto cuando sea relevante y pueda aportar información adicional.

Cada conversación se ha estudiado detenidamente y se han marcado las palabras clasificadas como coloquiales y malsonantes. Para cada vocablo señalado se ha recogido la palabra en su contexto, la referencia a la definición en el diccionario y un comentario general. Esta ficha es la base para el análisis discursivo del corpus. Además, se ha hecho una clasificación de las palabras según sus respectivas clases de palabras. El análisis se concentra

\footnotetext{
${ }^{4}$ http://www.rae.es/
} 
entonces en dos aspectos de las palabras registradas: la forma y la función pragmática o discursiva. Para clasificar las palabras según su forma, se toma en cuenta el contexto, así se reconoce la relación entre la forma y la función pragmática de las palabras.

La clasificación de las palabras según su función pragmática se ha hecho tomando en cuenta las principales funciones pragmáticas presentadas en la sección de definiciones y limitaciones. Además, en el proceso de clasificación también se ha evaluado si hay categorías que no han sido cubiertas por la teoría consultada y que se deberían elaborar.

\section{Resultados}

Los resultados se presentan en tres apartados independientes. En el primer apartado se presenta el inventario de las palabras coloquiales y malsonantes según la forma registrada. En el segundo apartado se analizan las palabras en su contexto, es decir la función pragmática de las palabras. En el tercer apartado se comparan los resultados y finalmente, se concluye con una discusión de los resultados.

\subsection{La forma de las palabras coloquiales y malsonantes}

\subsubsection{Sustantivos}

La clase de palabras que recoge más ejemplos de palabras coloquiales y malsonantes es el sustantivo. En la conversación MALCE2-3A de Madrid se han registrado 13 sustantivos diferentes, de los que nueve son sustantivos que se refieren a personas y se usan como vocativos, insultos o apelativos. Los cuatro sustantivos restantes son coño, hostia, mazo y pelas, como podemos ver en los ejemplos:

(1) MALCE2J01: $\quad$ y dice. dice (nombre) tiene coño y tetas como tú (1.153)

(2) MALCE2G09: $\quad$ que si que te doy una hooostia y te mando a. eh\(1. 102)

(3) MALCE2J01: $\quad$ tío me molan mazo los pantalones (1. 60)

(4) MALCE2J01: al mes soon dos mil quinientas pelas (1. 197)

Como se aprecia en los ejemplos, coño y hostia funcionan como sustantivos en este contexto, pero ambas palabras también se emplean como interjecciones en la misma conversación. Mazo no se registra en el DRAE en su uso informal, la definición que más se acerca al significado informal es " 3 . m. Conjunto abundante de papeles u otras cosas atadas o unidas formando grupo." En el Diccionario del argot, Sanmartín Sáez dice "El mazo (...) se utiliza en el habla informal para designar una gran cantidad de algo." (Sanmartín Sáez 2003, 552). Sin embargo, una búsqueda de mazo en la totalidad del corpus COLAm de Madrid indica un uso variado de mazo, no solo como sustantivo, pero más frecuentemente como adverbio modificando adjetivos o verbos cumpliendo las mismas funciones que mисhо у тиy. En (3) mazo se podría clasificar tanto como sustantivo de cantidad (me molan un montón) o como adverbio (me molan mucho), pero siguiendo la definición del diccionario, se ha elegido clasificar mazo como sustantivo. 
Al igual que en Madrid, en la conversación BABAS4-1-1 de Buenos Aires, la mayoría de los sustantivos son palabras que se refieren a personas. Los tres sustantivos que no se refieren a personas son culo, manón y paja, como podemos ver en los ejemplos:

(5) BABAS4J02: $\quad$ bueno pero a mí sí porque ... tiene un culo así de grande (1. 49)

(6) BABAS4J01: $\quad$ que se vayan a un manón (1. 99)

(7) BABAS4J01: $\quad$ es una paja] (1.97)

La categoría de sustantivos es la más frecuente en la conversación SCAWM4-03 de Santiago de Chile también, pero hay menos ejemplos de sustantivos que se refieren a personas. Debido al material limitado de estudio, puede ser que sea solo una coincidencia que la conversación no contenga muchos ejemplos de esta clase de sustantivos. Los sustantivos registrados en Santiago de Chile son cagada, huevada, onda y mierda, como indican los ejemplos:

(8) SCAWM4g12: $\quad$ aahh (xxx) para la cagada mierda (1.75)

(9) SCAWM4g13: $\quad$ mira la huevada flaca (1. 92)

(10) SCAWM4g13: no que onda (1.68)

Si comparamos los sustantivos coloquiales y malsonantes registrados en las tres conversaciones, vemos que no coinciden ninguno de los sustantivos.

\subsubsection{Verbos}

En Madrid se han registrado dos verbos con un significado que se puede clasificar como coloquial: molar y pirar. En (3) arriba podemos ver como el verbo molar se usa con el sustantivo mazo para formar la construcción "me molan mazo", mientras en (11) vemos el ejemplo de pirar usado como pronominal:

(11) MALCE2G03: $\quad$ se pira él (1.73)

Ambos verbos, molar y pirar provienen del caló, una fuente importante de palabras coloquiales en el español peninsular.

En Buenos Aires aparecen cinco verbos diferentes: bancar, cagar, chapar, chupar y joder. El verbo bancar se usa como pronominal:

(12) BABAS4J01: $\quad$ porque no me banco la gente así no me la banco si es falsa y careta (1. 104)

Según el Diccionario del español de Argentina, 'bancar' es una expresión juvenil que significa "soportar a una persona o situación que resulta molesta o desagradable", y se usa frecuentemente en frases negativas, tal como en (12).

En Santiago de Chile solo se ha registrado el verbo joder, un verbo que también hemos encontrado en Buenos Aires:

(13) SCAWM4g12: $\quad$ se jodió el trabajo (1. 79) 
En comparación con el uso en Buenos Aires, vemos que el significado del verbo joder es un poco diferente, ya que en (13) es usado con referencia a una cosa, mientras en (14) se refiere a una acción realizada por una persona:

(14) BABAS4J02: $\quad$ no no en serio o o. . . me estás jodiendo

\subsubsection{Adjetivos}

El único adjetivo registrado en Madrid es 'puto' y el femenino 'puta', que en ambos ejemplos se usa para modificar a un sustantivo:

(15) MALCE2J03: que voy con una puta camiseta negraa (1. 5)

(16) MALCE2G03: je je je puto (nombre) eh/ (1.106)

En Buenos Aires no se ha registrado ningún adjetivo, mientras en Santiago se ha usado el adjetivo flaite, de origen desconocido:

(17) SCAWM4g12: ah adónde se ha ido tan flaite (1.87)

Aunque no son muy comunes los adjetivos en estas tres conversaciones, es importante tomar en cuenta que los sustantivos usados como vocativos, insultos o apelativos en muchos casos son adjetivos sustantivados que incluyen una referencia a la cualidad de las personas. En el apartado 4.2 profundizamos más en estos sustantivos y sus funciones pragmáticas.

\subsubsection{Interjecciones}

En Madrid encontramos dos interjecciones: coño y jo. En (1) vimos que coño se usaba como sustantivo, mientras en (18) se emplea como interjección:

(18) MALCE2G09: no cuesta tanto eh/ que nooo coñoo (1. 96)

(19) MALCE2J01: $\quad$ sa grabao y sa escuchado en el jo (1. 120)

La conversación de Buenos Aires no incluye ningún ejemplo de interjecciones, mientras en Santiago de Chile encontramos la palabra malsonante puta usada como interjección:

(20) SCAWM4g12: puta momia saca las piernas (1.61)

\subsubsection{Otras expresiones}

No hay ejemplos de otras clases de palabras que sustantivos, verbos, adjetivos e interjecciones, pero sí hay algunas expresiones más o menos fijas que no se pueden clasificar dentro de una clase de palabras concreta. En Madrid se ha registrado la expresión por el culo:

(21) MALCE2J01: $\quad$ por el culo te la hinco (1. 117) 
Según DRAE, 'culo' no se clasifica como coloquial ni malsonante, pero por el contexto del ejemplo aquí se puede deducir que el mensaje es de insultar al interlocutor, lo cual indica que la expresión en sí tiene un significado malsonante.

En Buenos Aires, en cambio, se han registrado varias expresiones coloquiales. En (22) se usa la expresión ser de cuarta, que según el Diccionario fraseológico del habla argentina (FRA) significa 'Ser de poca categoría, despreciable':

(22) BABAS4J01: $\quad$ son de cuarta lo que hicieron (1. 12)

Otra expresión usada es chupar un huevo, que no aparece en ninguno de los diccionarios consultados, pero que se puede deducir que tenga un significado como "no me importa':

(23) BABAS4J01: tenés bueno me chupa un huevo que ellas hayan (1. 46)

La última expresión usada en Buenos Aires es dejar en banda, que según el FRA significa 'Dejar a alguien sin recurso, sin protección, sin compañía', un significado que coincide con el ejemplo:

(24) BABAS4J03: $\quad$ y te dejan en banda (1.65)

Además de las expresiones o locuciones de varias palabras, encontramos en el material de Buenos Aires el uso de tipo como un marcador discursivo:

(25) BABAS4J02: $\quad$ y me dice. $<\mathrm{I}>$ ay no sé </I > no y dice tipo. ay no sé y tipo se fue/ como te pue\% tipo (1. 76-77)

La única expresión encontrada en Santiago de Chile, es el insulto malsonante conche tu madre, usado como interjección:

(26) SCAWM4g12: $\quad$ qué (xxx) conche tu madre

\subsection{La función pragmática de las palabras coloquiales y malsonantes en su contexto}

Este artículo se basa en un material limitado, por lo que no tiene mucho valor hacer un estudio cuantitativo. Al contrario, al tratarse de conversaciones auténticas, el material es ideal para estudiar las funciones pragmáticas o discursivas de las palabras coloquiales y malsonantes en su contexto, y destacamos aquí los marcadores metadiscursivos, los conectores y los intensificadores.

\subsubsection{Los marcadores metadiscursivos}

Como se ha mencionado en el apartado 4.1, la mayoría de los sustantivos registrados en las conversaciones se refieren a personas, por lo que tienen una función expresivoapelativa. Estos sustantivos se usan sin artículo y su función pragmática puede variar de ser vocativos funcionando como controladores de contacto o apelativos que se dirigen directamente al interlocutor. Estos últimos pueden interpretarse como insultos, especialmente cuando son palabras malsonantes. 
En (27), MALCE2J03 usa el sustantivo femenino cerda en posición final del enunciado, una posición relacionada con la función de controlador de contacto. Se puede entonces interpretar como un vocativo en función de controlador de contacto o como un apelativo con el objetivo de insultar al interlocutor:

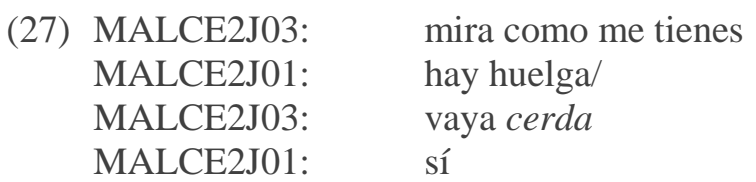

Los vocativos varían de los coloquiales tío o tía como en (28) a los insultos más ritualizados hijos de puta y cabrones en (29). Aunque hijo de puta y cabrones se consideran entre los insultos más graves en español (Gómez Molina 2003, 647), usados como vocativos como en el contexto de las grabaciones, se usan con el objetivo de reforzar la relación entre los hablantes (Jørgensen y Drange 2011).

(28) MALCE2J01: tío me molan mazo los pantalones

(29) MALCE2G03: $\quad$ síl eh eh eh hijos de puutaaa cabroones

Aparte de cerda, hijos de puta y cabrones en (27 y 29), solo encontramos los vocativos coloquiales macho, tronco, y tío/tía en el material de Madrid. Además de vocativos, encontramos sustantivos coloquiales como calificativos como en (30), donde vemos que el sustantivo pavas no tiene función de vocativo, sino que es usado para denominar a un grupo de personas clasificadas como ingenuas (Sanmartín Sáez 2003, 643):

(30) MALCE2J01: $\quad$ si me quiero enrollar con pavas ahora mismo

Como se ha indicado en 4.1, la mayoría de los sustantivos registrados en la conversación de Buenos Aires se usaron como apelativos o calificativos. El vocativo más usado es boludo/boluda, como en (31), un insulto que se refiere a la inteligencia de la persona:

\section{(31) BABAS4J02: $\quad$ s s son todas unas caretas boluda}

En posición final, la función principal del vocativo malsonante es reforzar las relaciones sociales. Encontramos el mismo vocativo malsonante en posición inicial, cuya función más bien es de tomar la palabra e iniciar una intervención:

(32) BABAS4J03: $\quad$ boluda son sus amigas ustedes

Otros vocativos en Buenos Aires son chico o chica y pelotuda, que se usan en mucho menor grado que boluda tanto en la conversación analizada como en todo el corpus COLAba. Los apelativos presentan una gran variedad, como caretas en (31), que en general son informales y no insultos reales, pero que muestran una gran creatividad en calificar a las personas en sentido negativo.

En Santiago de Chile también se aprecia el uso de sustantivos tanto como vocativos para llamar a una persona y como apelativos para hablar de las personas:

(33) SCAWM4g11: te voy a hacer una lista para que veas loco

(34) SCAWM4j02: no de los gallos de los cabros chicos 


\subsubsection{Conectores}

En el apartado anterior se han visto ejemplos de cómo se usan apelativos insultantes tanto en función de vocativos como de controladores de contacto. Cuando los vocativos se usan en posición inicial, funcionan también como conectores para tomar el turno o iniciar una intervención como en (35) donde vemos que MALCE2j01 introduce un nuevo tema e inicia su intervención con el vocativo tío:

(35) MALCE2G09: hace fresco parece que no pero MALCE2J01: tío estoy viciada a internet

Esta función de tomar la palabra también se realiza con expresiones malsonantes como en (36), donde coño inicia la intervención:

\section{(36) MALCE2G03: coño si este se ha cambiado el coche}

Un ejemplo de una expresión malsonante para tomar el turno también encontramos en la conversación de Santiago de Chile. Aquí 'puta' se puede interpretar tanto como un vocativo como una interjección utilizada para llamar la atención e iniciar el turno:

(37) SCAWM4g12: puta momia saca las piernas

En la conversación de Buenos Aires encontramos ejemplos de tipo en función de conector, tanto para tomar la palabra y explicar algo en (38) como para introducir una cita directa y concluir una intervención en (39):

(38) BABAS4J04: qué es internas

BABAS4J01: $\quad$ tipo las peleas entre amigas

(39) BABAS4J02: $\quad$ y me dice. $<$ I $>$ ay no sé $</$ I $>$ no y dice tipo. ay no sé y tipo se fue/ como te pue\% tipo

En general se ve que no se usan los mismos conectores en las distintas metrópolis, pero sería necesario abordar este tema en más profundidad antes de concluir en que las diferencias se deban a variaciones geolingüísticas entre las tres comunidades de habla.

\subsubsection{Intensificadores}

Por su naturaleza, las palabras malsonantes funcionan frecuentemente como intensificadores, en (40) vemos como 'puta' se usa para intensificar el sustantivo 'camiseta':

(40) MALCE2J03: que voy con una puta camiseta negraa

En las tres conversaciones también encontramos ejemplos de expresiones con elementos intensificadores que se usan para llamar la atención del interlocutor o expresar sus sentimientos. En (41) el hablante usa la palabra malsonante 'coño' para destacar su punto de vista, y el alargamiento de la vocal final subraya aún más la expresión:

(41) MALCE2G09: no cuesta tanto eh/ que nooo coñoo 
El hablante de Buenos Aires usa varias expresiones malsonantes para relacionarse con el interlocutor en (42):

(42) BABAS4J01: chico me voy a la mierda boluda me estás jodiendol

Tanto 'mierda' como 'joderse' como intensificadores encontramos igualmente en la conversación de Chile, como vemos en (43) y (44). En (43) vemos que 'mierda' se intensifica con el sinónimo 'la cagada':
(43) SCAWM4g12:
aahh (xxx) para la cagada mierda
(44) SCAWM4g12:
se jodió el trabajo

En (44) se usa el verbo malsonante joderse intensificando la actitud negativa del hablante hacia la situación en la que está el trabajo.

\subsection{Comparaciones}

En Madrid y Buenos Aires encontramos puta e hijo de puta usados como sustantivos, mientras en Madrid se usa puta como adjetivo también. En Santiago de Chile puta solo se ha registrado como una interjección en la conversación, y en el total de ejemplos del COLAs, el uso como interjección es el más frecuente.

En las tres comunidades de habla también encontramos variantes del verbo joder incluyendo la interjección jo registrada solo en Madrid. Una búsqueda en el corpus total de Madrid, muestra una gran cantidad de ejemplos de joder usado como verbo, lo que muestra que el verbo joder y la forma pronominal joderse se usan en las tres comunidades hispanohablantes estudiadas.

En las tres conversaciones analizadas aquí, variantes de la palabra huevo solo aparecen en Santiago de Chile y Buenos Aires. Sin embargo, una búsqueda en el corpus de Madrid muestra una variedad de expresiones que incluyen esta palabra, así que huevo y sus variantes también son comunes en las tres metrópolis.

Otro vocablo que solo se ha encontrado en las conversaciones analizadas de Santiago de Chile y Buenos Aires, es cagar y variantes de este verbo. Al igual que en los otros ejemplos mencionados aquí, variantes de cagar aparecen en el corpus COLAm de Madrid.

\section{Conclusión}

El objetivo del presente artículo ha sido analizar y comparar el uso de palabras coloquiales y malsonantes en tres comunidades de habla hispanohablantes. Se han comparado tres conversaciones informales auténticas, y es importante tener en mente que el tema de las conversaciones puede haber influido en las expresiones coloquiales y malsonantes que se hayan registrado en las conversaciones. Algunas expresiones son muy frecuentes en general y también en las conversaciones elegidas, mientras otras aparecen según el tema de conversación.

Al comparar la forma de las palabras, se ha visto que el sustantivo es la clase de palabras que registra la mayor cantidad de palabras coloquiales y malsonantes en las tres conversaciones. Sin embargo, al comparar los sustantivos coloquiales y malsonantes en las tres conversaciones, no coinciden ninguno de los sustantivos. Lo que sí coincide, es el uso de 
los sustantivos coloquiales y malsonantes; principalmente se usan como vocativos o apelativos. Así se puede concluir que el uso de sustantivos coloquiales y malsonantes en función de vocativos y apelativos es frecuente en las tres comunidades estudiadas, pero que las palabras usadas son diferentes.

En general, las expresiones coloquiales son más abundantes y más variadas que las expresiones malsonantes en las conversaciones. Esto indica que las expresiones coloquiales son más locales, mientras las principales expresiones malsonantes son similares en las tres variedades del español.

La comparación entre los conectores indica que hay variaciones en el uso de conectores en las conversaciones coloquiales. Este aspecto se podría estudiar en mayor profundidad, incluyendo conectores no específicamente coloquiales ya que aparentemente hay variedad en los conectores usados para tomar el turno, introducir un tema, etc.

En relación con los intensificadores, hay más similitudes entre las tres conversaciones, debido a que éstos principalmente son palabras malsonantes. Al encontrar una mayor coincidencia en las expresiones malsonantes en las tres comunidades, se puede deducir que en esta área hay más similitudes que en las palabras coloquiales.

Las implicaciones de estas conclusiones son que existe un sistema común, que incluye los elementos formales, mientras los elementos informales como los marcadores discursivos y la creatividad léxica del registro informal son diferentes. El sistema común también incluye aspectos culturales que se manifiestan en los temas tabú, resultando en que las expresiones malsonantes son parecidas en las tres comunidades de habla.

\section{Signos de transcripción}

$\begin{array}{ll}(\mathrm{xxx}) & - \text { Habla poco clara } \\ (\text { nombre }) & \text { - Nombre anonimizado } \\ \% & \text { - Palabra interrumpida } \\ / & - \text { Entonación ascendente de pregunta } \\ \mid & - \text { Entonación ascendente de pregunta } \\ \cdot & - \text { Pausa de un segundo } \\ \langle\text { I }\rangle \quad\langle/ \mathrm{I}\rangle & \text { - Imitación }\end{array}$

\section{Bibliografía}

Briz Gómez, Antonio. 2001. El español coloquial en la conversación. Esbozo de pragmagramática. 2. ${ }^{\mathrm{a}}$ ed. Barcelona: Editorial Ariel.

Calsamiglia Blancafort, Helena, y Amparo Tusón Valls. 2007. Las cosas del decir. Manual de análisis del discurso. 2. ${ }^{a}$ ed. Barcelona: Ariel Lingüística.

Calvo Shadid, Annette. 2009. «Análisis sociolingüístico sobre el tabú sexual en el español de Costa Rica». Bergen: Universidad de Bergen. 
Corpus Oral de Lenguaje Adolescente (COLA), Corpus disponible en: http://colam.org/indexespannol.html

Drange, Eli-Marie D., Ingrid Kristine Hasund, y Anna-Brita Stenström. 2014. «"Your mum!” Teenagers' swearing by mother in English, Spanish and Norwegian.» International Journal of Corpus Linguistics 19 (1): 29-59.

Gómez Molina, José Ramón. 2003. «El insulto: una aproximación sociolingüística». En Lengua, variación y contexto: estudios dedicados a Humberto López Morales, 2:63953. Madrid: Arco/Libros.

Hasund, Ingrid Kristine, Eli-Marie Drange, y Anna-Brita Stenström. 2014. «The pragmatic functions of swearing by mother in English, Spanish and Norwegian teenage talk». En Swearing in the Nordic Countries, 11-35. Sprognævnets konferenceserie 2. Copenhagen: Dansk Sprognavn.

Hernández Campoy, Juan Manuel. 1993. «Dialectología tradicional, sociolingüística laboviana y geolingüística trudgilliana: tres aproximaciones al estudio de la variación». https://doi.org/10.14198/ELUA1993.9.08.

Jørgensen, Annette Myre. 2007. «Cola: Un corpus oral de lenguaje adolescente». En Discurso y oralidad. Homenaje al profesor José Jesús de Bustos Tovar, 1:225-34. Madrid: Arco/Libros.

Jørgensen, Annette Myre, y Eli-Marie D. Drange. 2011. «La lengua juvenil de las metrópolis Madrid y Santiago de Chile». Arena Romanistica 9: 74-94.

Ljung, Magnus. 2011. Swearing: a cross-cultural linguistic study. Houndmills: Palgrave Macmillan.

Malaver, Irania, y José Antonio Samper Padilla. 2017. «Estudio de la /d/ intervocálica en los corpus PRESEEA». Boletín de Filología 51 (2): 325-45.

Moliner, María. 2007. Diccionario de uso del español. 3. a ed. Madrid: Gredos.

Moreno Fernández, Francisco Moreno. 2005. «Corpus Para El Estudio Del Español En Su Variación Geográfica Y Social. El Corpus “Preseea”». Oralia 8 (octubre): 123-39.

Pons Bordería, Salvador Pons, y Leonor Ruiz Ruiz Gurillo. 2005. «Corpus Para El Estudio De La Conversación Coloquial. El Corpus Val.es.co. (valencia. Español Coloquial)». Oralia 8 (octubre): 243-63.

PRESEEA, información disponible en: http://preseea.linguas.net/Metodolog\%C3\%ADa.aspx

Real Academia Española. Diccionario de la lengua española. 2017. ${ }^{\text {a }}$ ed. Madrid: Real Academia Española. http://www.rae.es/.

Sanmartín Sáez, Julia. 2003. Diccionario de argot. 4. ${ }^{\text {a }}$ ed. Madrid: Espasa Calpe.

Seco, Manuel. 2003. Estudios de lexicografía española. 2. ${ }^{a}$ ed. Madrid: Gredos.

Stenström, Anna-Brita. 2006. «Taboo words in teenage talk: London and Madrid girls" conversations compared.» Spanish in Context 3 (1): 115-38. 